\title{
Analysis and Discussion of Safety Management in Urban Rail Transit Operation
}

\author{
Hongzhao Zhang ${ }^{1, \mathrm{a}}$ \\ 'Jiangxi Vocational Technical College Of Industry \& Trade, Jiangxi, Nanchang, 330038 \\ a739960103@qq.com
}

\begin{abstract}
Urban rail transit industry is a specific and special service industry, what it needs is a safe management. Only when ensuring the safety of its operation, can we better improve the quality of the track operation. This paper is a comprehensive discussion on the importance of safety management, risk problems, measures and significance. The purpose is to improve the safety of the urban rail transit operation and management, and promote the development of the urban rail transit industry.
\end{abstract}

Keywords: Operation and management, urban rail transit, safety

\section{安全管理在城市轨道交通运营中的分析与探讨}

张洪照 ${ }^{1, \text { a }}$

\author{
1 江西工业贸易职业技术学院 江西 南昌 330103 \\ ${ }^{a} 739960103 @ q q . c o m$
}

摘要:

城市轨道交通行业是一个具体特殊性的服务行业, 它需要的是一个安全的管理。只有在确保其运营的安全 下，才能够更好的提高轨道运行的质量。本文就是从城市轨道交通运营安全管理的重要性、出现的分险问 题、措施以及意义这四个方面进行全面的探讨。目的就是提高城市轨道交通运营管理的安全, 促进城市轨道 交通行业的发展。

关键词：改革运营管理；城市轨道交通；安全性

\section{1. 引言}

城市轨道的交通网络系统在整个城市的交通网 络中起着重要的作用, 它决定着整个的交通运输网 络上运行状况。这也与生活里的每户人家和企业的 等的出行方式紧密联系在一起, 所以说提高一个城 市轨道交通运营管理的安全性是非常有必要的。如 果城市轨道的交通运营的不好的话, 就可能导致城 市交通的拥堵, 甚至可能出现城市道路一片混乱的 场景。总而言之, 城市轨道交通的发展将会影响着 城市许多行业未来的发展状况。

\section{2. 城市轨道交通运营安全管理的重要性探析}

如今，我国的经济飞速发展，导致我国城市轨 道运输业的要求也变得更高。又因为科学技术的进 步, 加上部分的现代化技术运用在城市轨道交通运 营安全管理的过程中，使得目前的交通网络发展越 来越复杂化。这样带给了管理工作人员许多的困难, 在城市轨道交通的运行中十分容易出现某些安全方 面的问题，最终是的整个的交通网络都无法正常的 运转。城市的轨道交通运输是否处于平稳的运行, 影响着该城市发展的稳定性，与此同时，它也影响 着居民的整个的生活水平如何。如果无法好好的对 城市轨道实行高效的管理，就会对城市的经济以及 其它方面造成损害，并且这些损失也是不能够用数 
字估量出来的。所以说, 对城市轨道交通运营管理 进行全面的研究是一个非常正确的选择。

\section{1. 影响着整个行业与社会的稳定性}

最近几十年以来, 我国的交通事故发生频繁, 尤其是城市轨道的运输方面。城市轨道的运输对每 个行业而言, 都是一个不能缺少的作用因素。然而, 却因为城市轨道运输的问题频繁发生造成人们的生 产与生活发展缓慢，更严重点就是造成交通事故、 人员的受伤与死亡。因为这种种的损失都是无法用 经济来衡量的, 所以人们就对城市轨道交通运输的 安全性提出了进一步的高要求。在这期间, 与交通 运输安全有关的技术都有了很大的发展, 众多城市 轨道交通运输的设备在运行中遇到的问题也被解决 了, 并出现了专业的管理环节, 促进了我国的交通 网络安全的管理工作的改进与发展。城市轨道交通 运营道德安全管理, 对整个的城市轨道交通运输业 起着巨大的作用。

\section{2. 直接影响着城市轨道运输本身}

交通网络本身的安全性的检修是必不可少的一 个步骤, 但是这也是我们要去解决的技术问题。城 市轨道交通运输业对于安全方面的要求是很高的, 一旦出现交通事故就将会危害着整个行业。交通网 络与整个社会、城市的发展息息相关, 提高交通城 市轨道运输行业的安全技术是非常有必要的。因此, 要有效经营城市轨道的运行, 并加强管理, 防止发 生交通事故，进而达到保护交通网络安全的作用。 城市轨道交通网络的安全, 影响着城市轨道运输自 己本身。如果安全性得到提高, 就会促进城市轨道 运输业的发展, 反之则会阻碍其发展。

\section{3. 城市轨道交通企业一定要安全管理}

将严格的责任制作为保障, 制定一个完备的安 全保障制度; 提升、加强工作人员的安全意识是整 个安全工作的前提, 加强对安全教育的学习, 营造 一个 “安全在每个人心中” 的大环境。让每一个员 工都没有违反安全法则的想法, 不敢也不能去违反。 加强思想上的认知, 学习安全管理的理论知识, 提 高自我的责任感, 让所有乘客的意识到交通安全对 人们健康的重要性，高效的减少事故的出现。另外, 企业也应该做到实、细、严，监督要达到规范化、 制度化、常态化的效果。然而, 经过考察后也应该 严格的依据公司制度的奖惩制度去处理, 将有关安 全的正规的法规与企业的企业制度真实的被运用, 加强规则的惩罚性、刚硬性、严肃性, 进而将安全 的火苗被消灭在初始阶段。

\section{3. 城市轨道交通运营安全管理现出现的风险 问题}

最近几年，城市轨道交通运营安全的总体趋势 比较平稳, 但是也出现了许多的问题。本文从管理、 人员、环境和设施设备四个不同的方面进行全方位 的分析。

\section{1. 与管理相关的方面}

因为管理不妥当导致的管理运营分险大都 表现 在安全的保障工作与制度方面上的漏洞。比如, 安 远管理的不妥当, 领导阶层的责任感不强, 监察管 理能力弱, 员工失职等; 制度的不完善导致各管理 的责任界限划分不够确切、工作的态度不够端正等 一系列问题, 这些全都将会增加交通网络安全的分 险。

\section{2. 与人员相关的方面}

工作人员这一方面的风险都大多数来自于技术、 心理与生理。人员因为技术问题会出现的危险行为 大都是因乘客缺少安全意识和常识, 在遇到突发情 况时无法冷静的处理; 心理问题导致的分险有心理 的承受能力差、组织纪律不强、安全的意识薄弱等 分险; 生理问题导致的分险主要是列车的司机在身 体上出现缺陷，违规或者无证驾驶。而非工作人员 因技术问题造成的风险表现在乘客具备的安全知识 少而诱发的危险动作; 心理问题导致的分险则表现 在乘客在车运输的途中因心理问题引起的不安全事 件; 生理问题说的就是乘客自身身体健康问题导致 的突发情况。

\section{3. 与环境相关的方面}

城市轨道交通运营的安全受环境的影响重大, 其中的环境不仅仅只是外部环境还有内部的环境。 内部的环境往往指的是车站里的公共地方、设备与 管理的房间、各区间等领域的空气质量。这些外部 的环境指标很大程度上会造成乘客与工作的人员患 病, 也有很大的可能性使得交通运输的设备等无法 正常的运行。外部的环境指的是恶劣的天气状况以 及灾害等自然的环境, 也包括不稳定的社会环境。 自然的环境状况是自然界造成的、人们要想改变这 种状况往往是比较困难的, 比如暴雪、大暴雨、犋 风等对城市轨道交通的设施设备的损坏。不稳定的 社会环境有人为的排放对人体有害的气体、放火等 种种行为。

\section{4. 与设备设施相关的方面}

如今, 城市轨道的交通网络是一个很复杂的系 统, 包含着很多的设备, 其中有基电、车辆和信号 设备等。要想让交通在运输过程中得到安全保障必 
须要有一个安全的环境下, 安全的设施和设备就显 得十分重要了。不论故障的大小与否, 都会严重的 影响交通运营的秩序，甚至导致重大事故发生。所 以，必须要保证交通轨道运输的设施与设备的质量， 进而保证城市交通轨道能够安全的运行。

\section{4. 提高我国的城市轨道交通运营安全的管理 方法}

\section{1. 建立一个系统化的质量管理体系}

一个好的交通轨道管理体系对整个的城市轨道 交通的运营起着重要的作用。简单的说, 在目前的 交通管理下, 提高整个城市的轨道交通管理能够有 利于促进交通网络的安全性。并且, 现在的城市轨 道交通的管理工作的评判依据是其交通运行的安全 管理体系。这也有利于提高提高整个城市轨道交通 运营的安全管理水平，同时还能够促进工程的运行。 新时代下, 人们对城市的交通安全提出了更高的要 求, 这就提醒我们要主动的想办法和采取措施来提 高管理工作的水平, 于此同时, 这对各个企业未来 的发展起着重要的作用, 与企业的生存发展息息相 关。

\section{2. 提高其管理工作人员的素质}

建筑的工程大部分都是通过管理的工作人员而 完成的, 管理工作人员的综合素质的高低直接与整 个的交通网络运行质量相关。管理工作的中心就是 拥有能力强、素质高的管理工作人员, 他们的素质 高低与能力强弱决定着整个城市轨道运输的管理质 量。因此, 要想提升技术工作人员的专业能力, 就 得需要企业去制定一些规范的机制, 并且对相关的 工作人员实行定期或者不定期的实习培训, 不断的 巩固以前学过的有关专业方面的知识, 学需新的管 理技能。通过提升管理工作人员的综合素养, 可以 使工作人员的工作能力与技术更加的强。然而, 管 理工作人员的道德教育也是非常重要的, 让管理人 员清楚的认识带交通运营安全的重要性。在城市轨 道交通运行的途中不断地去增强自己的素养, 进而 保证运营安全放在第一位。此外, 还要根据实际情 况去制定制度，让工作人员意识到自己身上的责任。 这样子工作人员才可以真正的把责任感加入到运营 安全管理工作中来。最后, 还应该要有一个考核制 度, 这也是维持一个公司企业好形象的有用途径, 对每次工作后都做出及时的评价, 作为员工年终考 核的依据之一, 并和工资相挂钩。这样的话, 能够 让消费者评价工作人员的服务, 给出意见, 而且还 可以使工作人员树立一个强烈的服务意识, 最终在 一定程度上提高城市轨道交通运营的管理质量。

\section{3. 变革管理制度, 实行现代化的管理。}

作为当代的城市轨道运营安全管理者，他们应 一直把保护好交通网络安全视为自己的责任, 做好 一个优质的管理者的态度，不可以忽视任何出现或 者未来将会出现的问题, 甚至小问题也不能够被忽 视。当然, 在管理的过程中应该及时的将发现的问 题解决处理好, 把它当作是本企业发展的重大问题。 还有就是, 也应该及时更新设备, 加快升级, 以及 不断的对管理者的管理技术进行培训, 可以话能够 积极的引入外国的先进技术, 增强企业的能力。然 后, 对于城市轨道交通的工作者, 他们应树立一个 高品质的管理思想, 要改变自己以为的管理理念去 挖掘新的客户，但也不能够只是简简单单的追求客 户的数量, 而去忽略了要想办法提高自己的服务水 平。要以客户的体验感为核心点去制定一个合理、 有效的制度。

\section{4. 加强对安全的监督}

在管理方式与制度被确定的情况下，要想让员 工和乘客遵守该管理方式和制度, 就必须安排对应 的人员去监督其他工作人员。因此可知, 一个完整 的监督体系在城市轨道交通运营安全管理中起到的 作用有多大。所以, 企业也应加快一步的落实好监 督方面的工作，把监督的工作安排的明明白白。同 时, 用高标准去要求监督的工作人员, 对他们进行 专业化的培训, 确保每个工作人员都能够秉持公正 的原则做好自己的监督工作。然而, 企业的内部则 要做的依据相关的标准及时的监督队管理人员的工 作情况, 监督组还能够以创立一个有关全国的城市 轨道交通安全的交流的系统, 能够及时的与他人交 流工作经验和总结工作, 实现全国的交流与合作。 最后, 不定期的、经常性的检查城市轨道交通运营 的安全管理工作, 目的是更好的规避安全分险, 预 防因安全引起的交通事故。

\section{5. 城市轨道的交通安全的意义}

城市轨道交通安全的意义具体表现为以下几个 方面:

(1) 乘客的安全直接受城市轨道交通的安全管理 的影响。城市轨道交通运营安全管理不仅能够使乘 客在出行方面得到满足, 而且还能够使城市的交通 秩序变得良好提供保证。

(2) 城市轨道交通运营安全管理与交通运输业持 续发展需求相符合。曾经, 因为司机工作的失职、 乘客的应变能力不足、设备出现故障等原因导致发 生许多严重的交通事故, 所以, 一定要站在长远的 利益的角度去思考问题, 实行安全管理的条例。进 而才能够增强乘客的乘车安全意识、加强责任感等, 以保证我国的城市轨道交通行业的不断前进发展。 
(3) 提高城市轨道交通运输业的安全, 是提高收 入的有用途径之一。一是没有污染并且噪音小, 满 足社会对环保的要求; 二是方便、安全、满足出行 需求者的需求。

(4) 城市轨道交通的安全管理有利于新的技术在 交通运输行业的生产里得到运用。将质量变得标准 化、管理变得精准化、信息变得安全化、设备变得 机械化当作保证安全与发展以及加强管理的重要措 施，使得经济收益与企业的管理同时得到发展。

(5) 城市轨道交通运营的安全管理有利于保证乘 客的安全。乘客在乘坐车辆的时候, 不仅仅会因为 无法抗拒的外部环境而导致身体上或者心理上的损 伤。只有城市轨道交通的各个方面的安全性得到保 证, 乘客的生命安全就会有进一步的保障。

\section{6. 结论:}

城市轨道交通运营安全管理仍然还要面临着许 多的分险和困难, 要重视其发展的安全性, 促进城 市交通运输业的发展。运输业的发展直接影响着经 济的发展, 无论采取什么样的方式, 都要提高员工 的工作安全意识, 这样才能够更好的保障城市轨道 交通运营安全管理。当然, 还需要一个优秀的领导, 带领企业走向成功的道路, 并且积极主动的去学习 外来的新思想、新技术以及高科技的设备。另外, 严格的执行每一项的工作, 把每份责任都真正的融 入到人们的心里, 这样能够使城市轨道交通运输业 在一定程度上实现巨大的发展, 推动所有运营安全 管理的发展进程。

\section{REFERENCES}

[1] Li Kang, Zhang,Honghong. Study on Beijing Metro[J]. Inner Mongolia Highway and Transportation.2017(5) :54-58.

[2] Zhang,Hongxing. Discussion on the Safety Management System of Urban Rail Transit Operation[J]. Urban fast rail transit.2017(30):110113.

[3] Chen,Wenhui. Strengthen the construction and operation safety management of urban rail transit projects[J]. Building materials and decoration. 2018(19).

[4] Mo,Weiran. Discussion and Practice of Guangzhou Metro Operation and Management[J]. Guangdong University of Technology.2007.

[5] Chen,Rubai. On Traffic Operation Safety Management — Take Rail Transit Management as an Example[J]. Shanxi Agricultural Economy. 2018 (5): 137.

[6] Sun,Bo.Huang,Shuzhi.Xue,Liang. Standardization of Safety Management of Urban Rail Transit Operation
Enterprises[J]. value engineering .2017, 36(28): 34-36.

[7] Zhang, Hengye. Discussion on the safety management of urban rail transit operation[J].China Equipment Engineering.2021.

[8] Xu,Xin. Discussion on the management mode of the network operation of urban rail transit[J].Theory Research on Urban Construction (Electronic Edition).2017(22).

[9] Zhao,Kai. Discussion on the safety management of urban rail transit operation[J].Smart cities.2019(04).

[10] Zhu,Haiyan.Xia,Jun. The construction and practice of the professional talent training mode of urban rail transit operation and management[J].New Curriculum Research (journal).2016(05).

[11] Zhu,Huayi. Analysis of urban rail transit safety management mode[J].Smart cities.2016(05).

[12] Xu,Tiankun. Research on the theory and method of the safety risk assessment of the urban rail transit network operation[D].Beijing Jiaotong University. 2012.

[13] Sun,Bo.Song,Tianyong.Liu,Jiguang. Research on the Construction Plan of Production Safety Standardization System for Urban Rail Transit Operation Enterprises in Liaoning Provinces[J]. Technology wind.2017(05).

[14] Kang,Tingting. Integrated standardization of urban rail transit operation safety and occupational health and safety management system[J].Neijiang Science and Technology.2017(01).

[15] Jin,Huai.Zhang,Chengman.Ma,Xuemei.Zhong,Qia orong.Wu,Fengbo.Li,Junwei.Establishment of the safety risk technology management system for urban rail transit[J].Urban Express Rail Transit.2010 (01).

[16] Lv,Jia. Discuss the problems existing in the management of urban rail transit operation in China[J].Scientific and technological innovation and application.2016(22).

[17] Zhang,Zongzhi.Analysis of urban rail transit operation and management problems in China[J].Oriental corporate culture.2015(02). 